\title{
Khovanov homology of graph-links
}

\author{
Igor Nikonov
}

\begin{abstract}
We define Khovanov homology over $\mathbb{Z}_{2}$ for graph-links.
\end{abstract}

\section{Introduction}

Graph-links 4, 5] are combinatorial analog of classical and virtual links. "Diagrams" of graph-links are simple undirected labeled graphs and graph-links themself are equivalence classes of the graphs modulo formal Reidemeister moves. In knot theory graph-links appear as intersection graphs of rotating chord diagrams of links. It is known that intersection graph determines chord diagram uniquely up to mutations [2]. Thus any link invariant that does not distinguish mutant links is a candidate for an invariant of graph-links. For example, Alexander polynomial, Jones polynomial and HOMFLY are of such type. The problem is the equivalence relation of the graph-links can be too strong so there can exist two chord diagrams which have different values of the invariant but whose intersection graphs coincide as graph-links. So the problem of finding a natural construction that would extend the invariant from intersection graphs to graph-links is nontrivial. For Jones polynomial this problem was solved by D. Ilyutko and V. Manturov [4]. This article provides a solution for (odd) Khovanov homology with coefficients in $\mathbb{Z}_{2}$.

A theory analogous to the theory of graph-links was developed by L. Traldi and L. Zulli [7. Their approach (called looped interlacement graphs theory) is based on Gauss diagrams of knots instead of rotating chord diagrams. D. Ilyutko showed these two theories are closely related [3. So the construction of the Khovanov homology described below will provide as well (after the necessary reformulation) a definition of the Khovanov homology for the looped interlacement graphs.

Odd Khovanov homology of links was defined by P. Ozsváth, J. Rasmussen and Z. Szabó [6. J. Bloom proved that odd Khovanov homology is mutant invariant [1] and we can expect it to be extended to an invariant of graphlinks. In fact the construction of J. Bloom over $\mathbb{Z}_{2}$ can be directly tranfered to the graph-link theory. One can also consider this invariant as the ordinary Khovanov homology of graph-links because for links both homology theories coincide modulo 2. On the other hand, integer-valued Khovanov homology is not mutant invariant [8] so we don't anticipate any adaption of it for graph-links. 


\section{Graph-links}

Let $\mathrm{G}$ be an undirected graph without loops and multiple edges and $\mathcal{V}=\mathcal{V}(G)$ be the set of its vertices. We assume $G$ be a labeled graph, i.e. every vertex $v$ in $G$ is endowed with a pair $(a, \alpha)$ where $a \in\{0,1\}$ is the framing $\operatorname{fr}(v)$ of $v$ and $\alpha \in\{-1,1\}$ is the $\operatorname{sign} \operatorname{sgn}(v)$ of the vertex $v$.

Let us fix an enumeration of vertices of $G$. We define the adjacency matrix $A(G)=\left(a_{i j}\right)_{i, j=1, \ldots, n}$ over $\mathbb{Z}_{2}$ as follows: $a_{i j}=1$ if and only if the vertices $v_{i}$ and $v_{j}$ are adjacent, and $a_{i j}=0$ otherwise. Besides we set $a_{i i}=\operatorname{fr}\left(v_{i}\right)$.

Let $v \in \mathcal{V}$. The set of all vertices in $\mathcal{V}$ adjacent to $v$ is called the neighbourhood of the vertex $v$ and denoted $N(v)$.

Let us define the Reidemeister moves of labeled graphs.

$\Omega_{1}$. The first Reidemeister move is an addition/removal of an isolated vertex labeled $(0, \pm 1)$.

$\Omega_{2}$. The second Reidemeister move is an addition/removal of two adjacent (resp. nonadjacent) vertices having the labels $(1,-1)$ and $(1,1)$ (resp. $(0,-1)$ and $(0,1))$ and the same neighbourhoods.

$\Omega_{3}$. The third Reidemeister move is defined as follows. Let $u, v, w$ be three vertices of $G$ having the labels $(0,-1)$ and $u$ be adjacent only to $v$ and $w$. Then we only change the adjacency of $u$ with the vertices $t \in N(v) \backslash N(w) \cup N(w) \backslash N(v)$ (for other pairs of vertices we don't change their adjacency). In addition, we change the signs of $v$ and $w$ to ' +1 '. The inverse operation is also called the third Reidemeister move.

$\Omega_{4}$. The fourth Reidemeister move is defined as follows. We take two adjacent vertices $u$ labeled $(0, \alpha)$ and $v$ labeled $(0, \beta)$. Then we change the label of $u$ to $(0,-\beta)$ and the label of $v$ to $(0,-\alpha)$. Besides, we change the adjacency for each pair $(t, w)$ of vertices where $t \in N(u)$ and $w \in N(v) \backslash N(u)$ or $t \in N(v)$ and $w \in N(u) \backslash N(v)$.

$\Omega_{4}^{\prime}$. In this fourth move we take a vertex $v$ with the label $(1, \alpha)$. We change the adjacency for each pair $(t, w)$ of vertices where $t, w \in N(u)$. Besides, we change the sign of $v$ and the framing for each $w \in N(u)$.

Definition 1. A graph-link is the equivalence class of a simple labeled graph modulo $\Omega_{1}-\Omega_{4}^{\prime}$ moves.

\section{Khovanov homology of graph links}

Let $G$ be a simple labeled graph with $n$ vertices and $A=A(G)$ be its adjacency matrix. We now formulate mod 2 version of Bloom's construction of odd Khovanov homology. The reason why we use $\mathbb{Z}_{2}$ is the chain complex construction depends on the coefficients of the adjacency matrix $A$ and the matrix $A$ is defined over $\mathbb{Z}_{2}$.

Suppose $s \subset \mathcal{V}=\mathcal{V}(G)$. We shall call subsets of $\mathcal{V}$ as states. Define $G(s)$ to be the complete subgraph in $G$ with the set of vertices $s$ and denote $A(s)=$ 
$A(G(s))$. Consider the vector space

$$
V(s)=\mathbb{Z}_{2}<x_{1}, \ldots, x_{n} \mid r_{1}^{s}, \ldots, r_{n}^{s}>
$$

where relations $r_{1}^{s}, \ldots, r_{n}^{s}$ are given by the formula

$$
r_{i}^{s}=\left\{\begin{array}{cl}
x_{i}+\sum_{\left\{j \mid v_{j} \in s\right\}} a_{i j} x_{j}, & \text { if } v_{i} \notin s, \\
\sum_{\left\{j \mid v_{j} \in s\right\}} a_{i j} x_{j}, & \text { if } v_{i} \in s
\end{array}\right.
$$

The dimension of $V(s)$ is equal to corank $A(s)$.

There is a natural bijection between states $s \subset \mathcal{V}$ and vertices of the hypercube $\{0,1\}^{n}$ : the state $s$ corresponds to the vector $\left(\alpha_{1}, \ldots, \alpha_{n}\right)$ where $\alpha_{i}=0$ if $v_{i} \in s$ and $\operatorname{sgn}\left(v_{i}\right)=1$ or if $v_{i} \notin s$ and $\operatorname{sgn}\left(v_{i}\right)=-1$ and $\alpha=1$ otherwise. Every edge of the hypercube is of the type $s \rightarrow s \oplus i$ where $s \oplus i$ denotes $s \cup\left\{v_{i}\right\}$ if $v_{i} \notin s$ and $s \backslash\left\{v_{i}\right\}$ if $v_{i} \in s$. We orient the arrow so that $v_{i} \notin s$ if $\operatorname{sgn}\left(v_{i}\right)=-1$ and $v_{i} \in s$ if $\operatorname{sgn}\left(v_{i}\right)=1$.

We assign to every edge $s \rightarrow s \oplus i$ the map $\partial_{s \oplus i}^{s}: \bigwedge^{*} V(s) \rightarrow \bigwedge^{*} V(s \oplus i)$ of exterior algebras defined by the formula

$$
\partial_{s \oplus i}^{s}(u)=\left\{\begin{array}{cl}
x_{i} \wedge u & \text { if } x_{i}=0 \in V(s), \\
u & \text { if } x_{i} \neq 0 \in V(s) .
\end{array}\right.
$$

Consider the chain complex

$$
C(G)=\bigoplus_{s \subset \mathcal{V}} \bigwedge^{*} V(s)
$$

with differential

$$
\partial(u)=\sum_{\left\{s, s^{\prime} \subset \mathcal{V} \mid s \rightarrow s^{\prime}\right\}} \partial_{s^{\prime}}^{s}(u)
$$

Proposition 1. Chain complex $(C(G), \partial)$ is well defined.

Proof. We need to show that the maps $\partial_{s \oplus i}^{s}$ are well defined and that each 2-face in the hypercube of states is commutative.

Lemma 1. Let us consider a state $s$ and an index $i$ such that $v_{i} \notin s$. We can assume without loss of generality that $A(s \oplus i)=\left(\begin{array}{cc}A & a^{\top} \\ a & \alpha\end{array}\right)$ where $A=A(s)$. Then

1. $x_{i}=0 \in V(s)$ iff $\operatorname{rank} A=\operatorname{rank}\left(\begin{array}{c}A \\ a\end{array}\right)$;

2. $x_{i}=0 \in V(s \oplus i)$ iff $\operatorname{rank}\left(\begin{array}{c}A \\ a\end{array}\right)+1=\operatorname{rank}\left(\begin{array}{cc}A & a^{\top} \\ a & \alpha\end{array}\right)$. 
Proof. It follows from the relation $x_{i}=\sum_{\left\{j \mid v_{j} \in s\right\}} a_{i j} x_{j}$ that the equality $x_{i}=0 \in$ $V(s)$ means the vector $a$ depends on the rows of the adjacency matrix $A$. This leads to the first statement of the lemma.

The equality $x_{i}=0 \in V(s \oplus i)$ implies that the vector $\left(\begin{array}{ll}0 & 1\end{array}\right)$ depends on the rows of the matrix $A(s \oplus i)$. Then $\operatorname{rank}\left(\begin{array}{cc}A & a^{\top} \\ a & \alpha\end{array}\right)=\operatorname{rank}\left(\begin{array}{cc}A & a^{\top} \\ a & \alpha \\ 0 & 1\end{array}\right)$. But

$$
\operatorname{rank}\left(\begin{array}{cc}
A & a^{\top} \\
a & \alpha \\
0 & 1
\end{array}\right)=\operatorname{rank}\left(\begin{array}{cc}
A & 0 \\
a & 0 \\
0 & 1
\end{array}\right)=\operatorname{rank}\left(\begin{array}{c}
A \\
a
\end{array}\right)+1
$$

Lemma 2. For any symmetric matrix $A$ if $\operatorname{rank}\left(\begin{array}{cc}A & a^{\top} \\ a & \alpha\end{array}\right)=\operatorname{rank} A+1$ then $\operatorname{rank}\left(\begin{array}{c}A \\ a\end{array}\right)=\operatorname{rank} A$.

Proof. Assume that $\operatorname{rank}\left(\begin{array}{c}A \\ a\end{array}\right) \neq \operatorname{rank} A$. Then $\operatorname{rank}\left(\begin{array}{cc}A & a^{\top} \\ a & \alpha\end{array}\right)=\operatorname{rank}\left(\begin{array}{c}A \\ a\end{array}\right)$ and the vector $\left(\begin{array}{c}a^{\top} \\ \alpha\end{array}\right)$ depends on the columns of the matrix $\left(\begin{array}{c}A \\ a\end{array}\right)$. Then the vector $a^{\top}$ depends of the columns of $A$ and (after transposition) the vector $a$ depends on the rows of $A$. Hence, $\operatorname{rank} A=\operatorname{rank}\left(\begin{array}{c}A \\ a\end{array}\right)$ that contradicts to the initial assumption.

Corollary 1. For any state $s$ and any index $i$ we have

1. $\operatorname{dim} V(s \oplus i)=\operatorname{dim} V(s)+1$ iff $x_{i}=0 \in V(s)$ and $x_{i} \neq 0 \in V(s \oplus i)$;

2. $\operatorname{dim} V(s \oplus i)=\operatorname{dim} V(s)-1$ iff $x_{i} \neq 0 \in V(s)$ and $x_{i}=0 \in V(s \oplus i)$;

3. $\operatorname{dim} V(s \oplus i)=\operatorname{dim} V(s)$ iff $x_{i}=0 \in V(s)$ and $x_{i}=0 \in V(s \oplus i)$.

The case $x_{i} \neq 0 \in V(s)$ and $x_{i} \neq 0 \in V(s \oplus i)$ is impossible.

Proof. The statements of the corollary follow from Lemmas 112 and the fact $\operatorname{dim} V(s)=\operatorname{corank} A(s), \operatorname{dim} V(s \oplus i)=\operatorname{corank} A(s \oplus i)$.

We call the first two cases in the proposition even and the third case odd. From the definition of the differentials we have $\partial_{s \oplus i}^{s}=0$ in the odd case.

Correctness of chain maps.

Let us consider the map $\partial_{s \oplus i}^{s}: \bigwedge^{*} V(s) \rightarrow \bigwedge^{*} V(s \oplus i)$. We must check that the relations are mapped to relations, that is for any element $u$ and any index $j$ there exist elements $u_{k} \in V(s \oplus i)$ such that

$$
\partial_{s \oplus i}^{s}\left(r_{j}^{s} \wedge u\right)=\sum_{k} r_{k}^{s \oplus i} \wedge u_{k} \in V(s \oplus i) .
$$


For any $j$ we have $r_{j}^{s}=r_{j}^{s \oplus i}+\alpha_{j} x_{i}$ for some $\alpha_{j} \in \mathbb{Z}_{2}$. If $x_{i}=0 \in V(s \oplus i)$ then

$$
\partial_{s \oplus i}^{s}\left(r_{j}^{s} \wedge u\right)=r_{j}^{s} \wedge u^{\prime}=r_{j}^{s \oplus i} \wedge u^{\prime}+\alpha_{j} x_{i} \wedge u^{\prime}=r_{j}^{s \oplus i} \wedge u^{\prime}
$$

in $V(s \oplus i)$, where $u^{\prime}=u$ or $u^{\prime}=0$. If $x_{i} \neq 0 \in V(s \oplus i)$ then $x_{i}=0 \in V(s)$ and

$$
\partial_{s \oplus i}^{s}\left(r_{j}^{s} \wedge u\right)=x_{i} \wedge r_{j}^{s} \wedge u=x_{i} \wedge r_{j}^{s \oplus i} \wedge u+\alpha_{j} x_{i} \wedge x_{i} \wedge u=r_{j}^{s \oplus i} \wedge\left(x_{i} \wedge u\right) .
$$

In any case the map $\partial_{s \oplus i}^{s}$ is well defined.

Commutativity of 2-faces.

Every 2-face of hypercube looks like

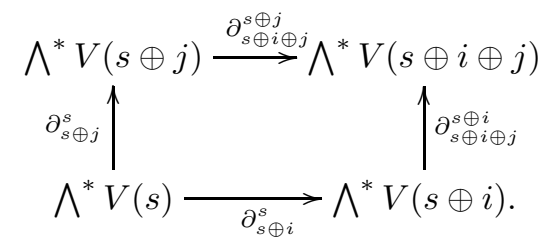

According to dimensions of spaces $V\left(s^{\prime}\right), s^{\prime}=s, s \oplus i, s \oplus j, s \oplus i \oplus j$ we have five types of diagrams without odd edges:

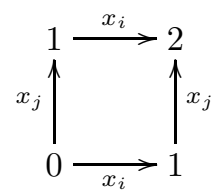

Type 1

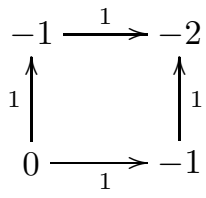

Type 2

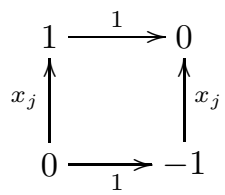

Type 3

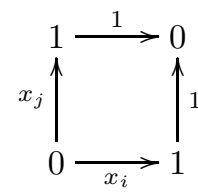

Type 4

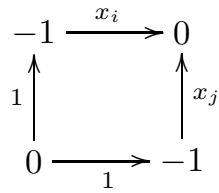

Type 5

Here the number at the place of the state $s^{\prime}$ is equal to $\operatorname{dim} V\left(s^{\prime}\right)-\operatorname{dim} V(s)=$ corank $A\left(s^{\prime}\right)-\operatorname{corank} A(s)$ and the label $z=1, x_{i}, x_{j}$ at the arrow for the map $\partial_{s^{\prime \prime}}^{s^{\prime}}$ means that $\partial_{s^{\prime \prime}}^{s^{\prime}}(u)=z \wedge u$.

2-faces of types 1,2,3 are obviously commutative. Any 2-face of type 4 is commutative because $x_{i}=x_{j}=0 \in V(s \oplus i \oplus j)$. For the face of type 5 we need to show that $x_{i}=x_{j} \in V(s \oplus i \oplus j)$. There are three possibilities.

1. $\operatorname{sgn}\left(v_{i}\right)=\operatorname{sgn}\left(v_{j}\right)=-1$. Then $v_{i}, v_{j} \in s \oplus i \oplus j$. Without loss of generality we can assume that $v_{i}$ and $v_{j}$ are the last vertices in $s \oplus i \oplus j$. The adjacency matrix $A(s \oplus i \oplus j)$ can be represented in the form

$$
\left(\begin{array}{ccc}
A & a^{\top} & b^{\top} \\
a & \alpha & \gamma \\
b & \gamma & \beta
\end{array}\right)
$$


where $A=A(s)$.

Since corank $A(s \oplus i)=\operatorname{corank} A(s)-1$ we have $x_{i}=0 \in V(s \oplus i)$. Then the rows of the matrix $A(s \oplus i)=\left(\begin{array}{cc}A & a^{\top} \\ a & \alpha\end{array}\right)$ generate the vector $\left(\begin{array}{ll}0 & 1\end{array}\right)$. Hence, the rows of the matrix $A(s \oplus i \oplus j)$ generate the vector $(01 \delta), \delta \in \mathbb{Z}_{2}$. If $\delta=0$ then $x_{i}=0 \in V(s \oplus i \oplus j)$ but this is not the case. So $\delta=1$ and we have the relation $x_{i}+x_{j}=0$ in $V(s \oplus i \oplus j)$.

2. $\operatorname{sgn}\left(v_{i}\right)=-1, \operatorname{sgn}\left(v_{j}\right)=1$. Then $v_{i} \in s \oplus i \oplus j$ and $v_{j} \notin s \oplus i \oplus j$. Assume that the adjacency matrix $A(s \oplus i)$ has the form (3) where $A=A(s \oplus j)$.

We have the equality $x_{i}=0 \in A(s \oplus i)$. It implies that the vector $\left(\begin{array}{lll}0 & 1 & 0\end{array}\right)$ is a linear combination of the rows of the adjacency matrix $A(s \oplus i)$. If the coefficient in the linear combination by the row $(b \gamma \beta)$ is zero then the rows of the matrix

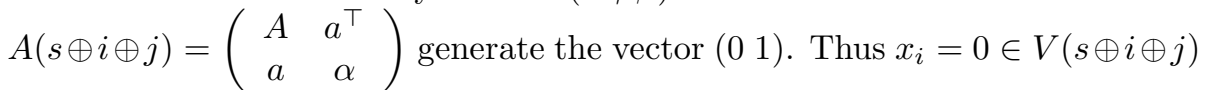
that leads to contradiction. Therefore the coefficient by $(b \gamma \beta)$ is 1 . Then the vector $(b \gamma+1)$ is generated by the rows of the matrix $A(s \oplus i \oplus j)$ that means $x_{i}+x_{j}=0 \in V(s \oplus i \oplus j)$.

3. $\operatorname{sgn}\left(v_{i}\right)=\operatorname{sgn}\left(v_{j}\right)=-1$. Then the matrix $A(s)$ looks like (3) where $A=A(s \oplus i \oplus j)$. The equality $x_{i}+x_{j}=0 \in V(s \oplus i \oplus j)$ corresponds to the relation vector $a+b$. Since $\operatorname{rank} A(s)=\operatorname{rank} A(s \oplus j)=\operatorname{rank}\left(\begin{array}{cc}A & a^{\top} \\ a & \alpha\end{array}\right)=$ $\operatorname{rank}\left(\begin{array}{ccc}A & a^{\top} & b^{\top} \\ a & \alpha & \gamma\end{array}\right)$ the last row in the matrix $A(s)$ is expressed as a linear combination of the other rows. If the coefficient by the row $(a \alpha \gamma)$ in the combination is zero then the vector $(b \gamma \beta)$ depends on the rows of the matrix ( $\left.\begin{array}{lll}A & a^{\top} & b^{\top}\end{array}\right)$ and the vector $b$ depends on the rows of the matrix $A$. Hence, $x_{j}=0 \in V(s \oplus i \oplus j)$ that is not true. Thus, the coefficients by $(a \alpha \gamma)$ is not zero so the vector $a+b$ is generated by the rows of the matrix $A$ that means $x_{i}+x_{j}=0 \in V(s \oplus i \oplus j)$.

Any diagram, which has odd edges in both upper-right and left-upper paths, is commutative because the map of any odd edge is zero. After this remark it remains only two nontrivial diagrams (up to symmetry between $i$ and $j$ ) with an odd edge:

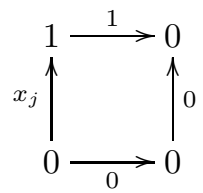

Type 6

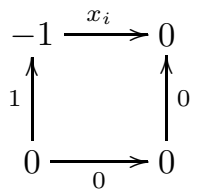

Type 7

The diagram of type 6 is commutative because $\operatorname{dim} V(s \oplus i)=\operatorname{dim} V(s \oplus i \oplus j)$ so $x_{j}=0 \in V(s \oplus i \oplus j)$.

For the commutativity of the diagram of type 7 we need to prove that $x_{i}=$ $0 \in V(s \oplus i \oplus j)$. Assume this is not the case. 
Let $\operatorname{sgn}\left(v_{i}\right)=\operatorname{sgn}\left(v_{j}\right)=-1$. Then the adjacency matrix looks like (3). Since $x_{j}=0 \in V(s \oplus i)$ we have $\operatorname{rank}\left(\begin{array}{cc}A & a^{\top} \\ a & \alpha \\ b & \gamma\end{array}\right)=\operatorname{rank}\left(\begin{array}{cc}A & a^{\top} \\ a & \alpha\end{array}\right)$. Then $b$ depends on the rows of $\left(\begin{array}{c}A \\ a\end{array}\right)$. But rank $\left(\begin{array}{c}A \\ a\end{array}\right)=\operatorname{rank} A$ hence $b$ depends on the rows of $A$. On the other hand, we have $x_{j} \neq 0 \in V(s)$ so $\operatorname{rank}\left(\begin{array}{c}A \\ b\end{array}\right) \neq$ $\operatorname{rank} A$. This is contradiction.

The other cases of sign arrangement can be treated in an analogous manner. This concludes the proof of Proposition 1.

Definition 2. We call the homology $\overline{\mathrm{Kh}}(G)$ of the complex $(C(G), \partial)$ the reduced (odd) Khovanov homology of the labeled simple graph $G$.

The main theorem of the article states that Khovanov homology is well defined for graph-links.

Theorem 1. Khovanov homology $\overline{\mathrm{Kh}}(G)$ is invariant under $\Omega_{1}-\Omega_{4}^{\prime}$ moves.

Proof. Let $G$ be a labeled graph and $\widetilde{G}$ be the graph obtained from $G$ by some Reidemeister move $\Omega_{1}-\Omega_{4}^{\prime}$.

Invariance under $\Omega_{1}$.

Let $\widetilde{G}$ be obtained from $G$ by an addition of an isolated labeled vertex $v$. The complex $C(\widetilde{G})$ is isomorphic to the product of complexes $C(G) \otimes C(v)$ where the complex $C(v)$ is equal to

$$
\mathbb{Z}_{2} \stackrel{x \wedge}{\longrightarrow} \bigwedge^{*} \mathbb{Z}_{2}\langle x\rangle
$$

if $\operatorname{sgn}(v)=-1$ and

$$
\bigwedge^{*} \mathbb{Z}_{2}\langle x\rangle \stackrel{x=0}{\longrightarrow} \mathbb{Z}_{2}
$$

if $\operatorname{sgn}(v)=1$. In any case $H_{*}(C(v))=\mathbb{Z}_{2} \cdot 1$, where $1 \in H_{0}(C(v))$ if $\operatorname{sgn}(v)=1$ and $1 \in H_{1}(C(v))$ if $\operatorname{sgn}(v)=-1$. Thus, we have

$$
\overline{\mathrm{Kh}}(\widetilde{G})=\overline{\mathrm{Kh}}(G) \otimes \overline{\mathrm{Kh}}(v) \cong \overline{\mathrm{Kh}}(G) .
$$

Invariance under $\Omega_{2}$.

Assume that we add the vertices $v$ and $w$ to get the graph $\widetilde{G}$ by $\Omega_{2}$ and $\operatorname{sgn}(v)=1, \operatorname{sgn}(w)=-1$. Without loss of generality we can write the adjacency matrix $A(\widetilde{G})$ in one of this two forms

$$
\left(\begin{array}{ccc}
0 & 0 & a^{\top} \\
0 & 0 & a^{\top} \\
a & a & A(G)
\end{array}\right) \quad \text { or } \quad\left(\begin{array}{ccc}
1 & 1 & a^{\top} \\
1 & 1 & a^{\top} \\
a & a & A(G)
\end{array}\right)
$$

In both cases for every state $s \in \mathcal{V}(G)$ we have the following equalities: corank $A(G(s))=$ $\operatorname{corank} A(\widetilde{G}(s))$, corank $A(\widetilde{G}(s \cup\{v\}))=\operatorname{corank} A(\widetilde{G}(s \cup\{w\}))=\operatorname{corank} A(\widetilde{G}(s \cup$ 
$\{v, w\}))-1$. These equalities define the type of the upper and left arrows of the complex $C(\widetilde{G})$ written in the form

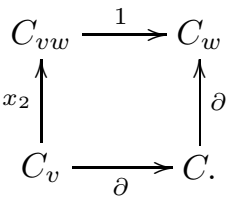

Here $C_{v}$ consists of the chains whose state contains $v$ and does not contain $w$; the complexes $C, C_{w}, C_{v w}$ are defined analogously.

For any state $s$ in $C_{v w}$ let us define a linear function $f: V(s) \rightarrow \mathbb{Z}_{2}$ by the formula $f\left(\sum_{i} \lambda_{i} x_{i}\right)=\lambda_{1}+\lambda_{2}$. The function $f$ is well defined because it equals to zero on any relation: $f\left(r_{i}^{s}\right)=a_{i 1}+a_{i 2}=0$ since $a_{i 1}=a_{i 2}$. Then $\Lambda^{*} V(s)=\Lambda^{*} \operatorname{ker} f \oplus x_{2} \Lambda^{*} V(s)$ and $C_{v w}=X \oplus x_{2} C_{v w}$. The subcomplex $X \rightarrow C_{w}$ is acyclic because the arrow is an isomorphism. Then the homology of $C(\widetilde{G})$ coincides with the homology of the quotient complex

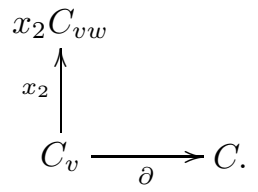

The quotient of the obtained complex by the subcomplex $C$ appears to be acyclic too. Thus $C(\widetilde{G})$ has the same homology as $C=C(G)$.

Invariance under $\Omega_{3}$.

Without loss of generality we can assume that the vertices $u, v, w$ in the third Reidemeister move have the indices $1,2,3$ in $\mathcal{V}(G)=\mathcal{V}(\widetilde{G})$. There are two variants of $\Omega_{3}$ depending adjacency of $v$ and $w$ and we consider the version where $v$ and $w$ are not adjacent. The proof for the other version is analogous. The adjacency matrices of $G$ and $\widetilde{G}$ looks like

$$
A(G)=\left(\begin{array}{cccc}
0 & 1 & 1 & 0^{\top} \\
1 & 0 & 0 & a^{\top} \\
1 & 0 & 0 & b^{\top} \\
0 & a & b & B
\end{array}\right), \quad A(\widetilde{G})=\left(\begin{array}{cccc}
0 & 0 & 0 & (a+b)^{\top} \\
0 & 0 & 0 & a^{\top} \\
0 & 0 & 0 & b^{\top} \\
a+b & a & b & B
\end{array}\right) .
$$

Denote $\widetilde{V}(s)=V(\widetilde{G}(s))$. Then for any $s \subset \mathcal{V}(G) \backslash\{u, v, w\}$ we have $V(s) \cong$ $\widetilde{V}(s), V(s \oplus v) \cong \widetilde{V}(s \oplus v), V(s \oplus w) \cong \widetilde{V}(s \oplus w), V(s \oplus v \oplus w) \cong \widetilde{V}(s \oplus u \oplus v) \cong$ $\widetilde{V}(s \oplus u \oplus w), V(s \oplus u \oplus v \oplus w) \cong \widetilde{V}(s \oplus u), V(s \oplus u \oplus v) \cong V(s \oplus u \oplus w) \cong V(s)$ and the correspondent isomorphisms of the exterior algebras are compatible with the differential. 
Consider complexes $C(G)$ and $C(\widetilde{G})$ in the form of cubes:
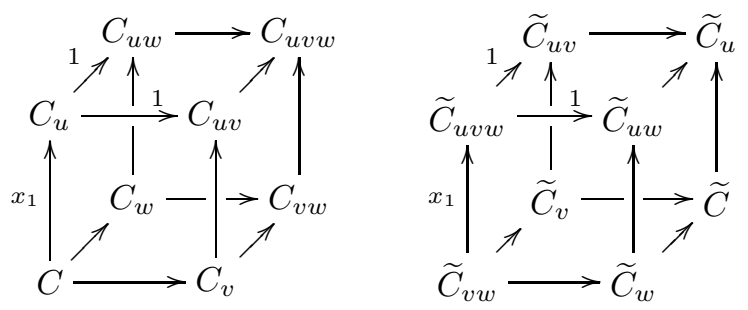

For any state $s$ in $C_{u}$ let us define a linear function $f: V(s) \rightarrow \mathbb{Z}_{2}$ by the formula $f\left(\sum_{i} \lambda_{i} x_{i}\right)=\lambda_{1}$. The function is well defined and there are decompositions $\bigwedge^{*} V(s)=\bigwedge^{*} \operatorname{ker} f \oplus x_{1} \bigwedge^{*} V(s)$ and $C_{u}=X \oplus x_{1} C_{u}$. Consider the following subcomplex

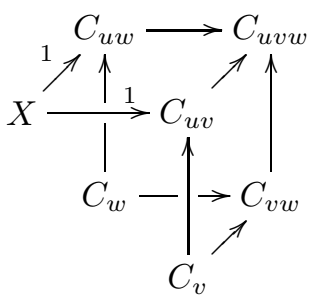

The quotient complex $C \rightarrow x_{1} C_{u}$ is acyclic so the homology of $C(G)$ is isomorphic to the homology of the subcomplex. This subcomplex contains the acyclic subcomplex $X \rightarrow \partial(X)$. The maps $X \rightarrow C_{u v}$ and $X \rightarrow C_{u w}$ are isomorphisms, so after factorization we obtain the complex

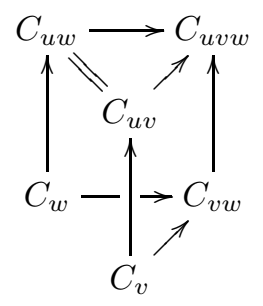

where the spaces $C_{v}$ and $C_{w}$ are identified.

The reasonings analogous to the reasonings above reduce $C(\widetilde{G})$ to the complex (for a state $s$ in $\widetilde{C}_{\text {uvw }}$ we should define the function $f: \widetilde{V}(s) \rightarrow \mathbb{Z}_{2}$ by the formula $\left.f\left(\sum_{i} \lambda_{i} x_{i}\right)=\lambda_{1}+\lambda_{2}+\lambda_{3}\right)$.

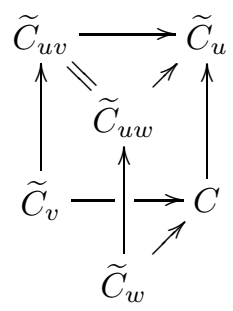


Both complexes are isomorphic to the complex $C_{v} \oplus C_{w} \rightarrow C \oplus C_{v w} \rightarrow C_{u v w}$. Thus $C(G)$ and $C(\widetilde{G})$ have the same homology.

Invariance under $\Omega_{4}$.

Let the vertices $u$ and $v$ of the move $\Omega_{4}$ have the indices $p$ and $q$ in $V(G)=$ $V(\widetilde{G})$. The coefficients of adjacency matrices of $A(G)=\left(a_{i j}\right)$ and $A(\widetilde{G})=\left(\widetilde{a}_{i j}\right)$ are connected by the formula

$$
\tilde{a}_{i j}=\left\{\begin{array}{cl}
a_{i j}+a_{i p} a_{j q}+a_{i q} a_{j p}, & \{i, j\} \cap\{p, q\}=\emptyset, \\
a_{i j}, & \{i, j\} \cap\{p, q\} \neq \emptyset .
\end{array}\right.
$$

Consider the map $\phi$ acting on the states by the formula

$$
\phi(s)=\left\{\begin{array}{cl}
s \cup\{u, v\}, & \{u, v\} \cap s=\emptyset, \\
s \backslash\{u, v\}, & \{u, v\} \cap s=\{u, v\}, \\
s, & \{u, v\} \cap s \neq \emptyset,\{u, v\}
\end{array}\right.
$$

together with the linear maps $\Phi: V(s) \rightarrow \widetilde{V}(\phi(s))$ defined by the formula

$$
\Phi\left(x_{i}\right)= \begin{cases}x_{i}, & i \neq p, q, \\ x_{q}, & i=p, \\ x_{p}, & i=q .\end{cases}
$$

One can check that the map $\Phi$ defines an isomophism of the vector spaces and after the natural extension to an isomorphism of external algebras $\wedge^{*} V(s) \rightarrow$ $\bigwedge^{*} \widetilde{V}(\phi(s))$ for each state $s$ it determines an isomophism of the graded linear spaces $\Phi: C(G) \rightarrow C(\widetilde{G})$. $\Phi$ is a chain map. Thus the complexes $C(G)$ and $C(\widetilde{G})$ are isomorphic as well as their homology.

Invariance under $\Omega_{4}^{\prime}$.

Let the vertex $v$ of the move $\Omega_{4}^{\prime}$ have the index $p$. The coefficients of adjacency matrices of $A(G)=\left(a_{i j}\right)$ and $A(\widetilde{G})=\left(\widetilde{a}_{i j}\right)$ are connected by the formula

$$
\widetilde{a}_{i j}=\left\{\begin{array}{cl}
a_{i j}+a_{i p} a_{j p}, & i, j \neq p \\
a_{i p}, & j=p \\
a_{p j}, & i=p
\end{array}\right.
$$

Consider the map $\phi: C(G) \rightarrow C(\widetilde{G})$ which acts on the states by the formula

$$
\phi(s)=s \oplus p
$$

and the linear maps $\Phi: V(s) \rightarrow \widetilde{V}(\phi(s))$ defined on the generators by the formula $\Phi\left(x_{i}\right)=x_{i}, 1 \leq i \leq n$. Then $\Phi$ induces a well defined isomorphism of the complexes $C(G)$ and $C(\widetilde{G})$ so the homologies of the complexes coincide.

Corollary 2. Khovanov homology $\overline{\operatorname{Kh}}(G)$ is an invariant of graph-links. 


\section{Jones polynomial of graph-knots and Khovanov homology}

Let $G$ be a simple labeled graph. The complex $C(G)$ has two grading: the homological grading $M_{0}$ and the algebraic grading deg of graded algebras $\wedge^{*} V(s)$. The differential is not homogeneous with respect to deg but it is compatible with the grading $Q_{0}$ where for element any $u \in \Lambda^{r}(s)$ we define $Q_{0}(u)=$ $\operatorname{dim}_{\mathbb{Z}_{2}} V(s)-2 r+M_{0}(s)$. The differential increases the grading $M_{0}$ and leaves the grading $Q_{0}$ unchanged. Then

$$
\overline{\mathrm{Kh}}(G)=\bigoplus_{m, q \in \mathbb{Z}} \overline{\mathrm{Kh}}(G)_{(m, q)} .
$$

The following definition is due to Ilyutko and Manturov [4].

Definition 3. Let $G$ be a simple labeled graph with $n$ vertices. The Kauffman bracket polynomial of $G$ is defined as

$$
\langle G\rangle(a)=\sum_{s \subset \mathcal{V}(G)} a^{\alpha(s)-\beta(s)}\left(-a^{2}-a^{-2}\right)^{\operatorname{corank} A(s)},
$$

where $\alpha(s)=\#\{v \in s \mid \operatorname{sgn}(v)=-1\}+\#\{v \notin s \mid \operatorname{sgn}(v)=1\}$ and $\beta(s)=$ $n-\alpha(s)$.

It appears that the Poincaré polynomial of the bigraded Khovanov homology of the graph $G$ coincides with its Kauffman bracket up to shift of the gradings.

\section{Theorem 2.}

$$
\sum_{m, q \in \mathbb{Z}}(-1)^{m} \operatorname{dim}_{\mathbb{Z}_{2}} \overline{\operatorname{Kh}}(G)_{(m, q)} \cdot t^{q}=\left(i t^{1 / 2}\right)^{-n}\langle G\rangle\left(i t^{1 / 2}\right) .
$$

Proof. The left term of the equality coincides with the Poincaré polynomial of the chain complex $C(G)$. Each state $s \subset \mathcal{V}(G)$ has homological grading $\alpha(s)$ and the corresponding chain space $\bigwedge^{*} V(s)$ contributes $(-1)^{\alpha(s)}\left(t+t^{-1}\right)^{\operatorname{dim} V(s)} t^{\alpha(s)}$ to the Poincaré polynomial. Since $\operatorname{dim} V(s)=$ corank $A(s)$ and $\alpha(s)=\frac{1}{2}(\alpha(s)-$ $\beta(s)-n)$ the sum over all states yields the right term of the equality.

The gradings $M_{0}, Q_{0}$ of the Khovanov complex are not preserved by the Reidemeister moves as shown in the following table.

\begin{tabular}{|c|c|c|}
\hline & $M_{0}$ & $Q_{0}$ \\
\hline$\Omega_{1}^{+}$ & 0 & -1 \\
$\Omega_{1}^{-}$ & 1 & 1 \\
$\Omega_{2}$ & 1 & 0 \\
$\Omega_{3}$ & 0 & 0 \\
$\Omega_{4}$ & 0 & 0 \\
$\Omega_{4}^{\prime}$ & 0 & 0 \\
\hline
\end{tabular}


Here $\Omega_{1}^{ \pm}$denotes the adding an isolated vertex with the label \pm 1 and $\Omega_{2}$ denotes the adding two vertices. The entries of the cells are the shifts of the gradings after the corresponding move.

Thus the groups $\overline{\operatorname{Kh}}(G)_{(m, q)}$ are not invariants of graph-links. Nevertheless we can normalize the gradings for the graph-knots.

Definition 4 (4). A graph-link $G$ is called a graph-knots if corank $(A(G)+E)=$ 0 where $E$ is the identity matrix.

The condition corank $(A(G)+E)=0$ survives after Reidemeister moves so the definition is correct.

Definition 5 (4). Writhe number of a graph $G$ with $n$ vertices is the sum

$$
w(G)=\sum_{i=1}^{n}(-1)^{\operatorname{corank} B_{i}(G)} \operatorname{sgn} v_{i}
$$

where $B_{i}(G)=A(G)+E+E_{i i}$ and the only non-zero element of $E_{i i}$ lies in the $i$-th row and $i$-th column.

Writhe number is invariant under the moves $\Omega_{2}-\Omega_{4}$. The move $\Omega_{1}^{ \pm}$changes the writhe number by $\mp 1$. Using this fact and the table (4) we construct two gradings which are invariant under the Reidemeister moves

$$
M=M_{0}-\frac{n(G)+w(G)}{2}, \quad Q=Q_{0}+\frac{n(G)+3 w(G)}{2}
$$

where $n(G)$ is the number of vertices in $G$.

Let us denote $\overline{\mathrm{Kh}}_{m, q}(G)$ to be the homogeneous part of $\overline{\mathrm{Kh}}(G)$ with the gradings $M=m$ and $Q=q$. The groups $\overline{\mathrm{Kh}}_{m, q}(G)$ are invariants of graphknots.

Writhe number allows to introduce another invariant of graph-knots.

Definition 6 (4). Jones polynomial of a graph-knot $G$ is defined as

$$
X(G)(a)=(-a)^{-3 w(G)}\langle G\rangle(a) .
$$

Theorem 2 leads to the following statement.

Theorem 3. The bigraded Khovanov homology of graph-knots $\overline{\mathrm{Kh}}_{m, q}(G)$ categorifies the Jones polynomial in the sense that

$$
\sum_{m, q \in \mathbb{Z}}(-1)^{m} \operatorname{dim}_{\mathbb{Z}_{2}} \overline{\operatorname{Kh}}_{m, q}(G) \cdot t^{q}=X(G)\left(i t^{1 / 2}\right) .
$$

The author is grateful to V. Manturov and D. Ilyutko for useful discussions. 


\section{References}

[1] J. Bloom, Odd khovanov homology is mutation invariant // Math. Res. Lett. 17(1) (2010), 1-10.

[2] S. Chmutov, S. Lando, Mutant knots and intersection graphs // Alg. Geom. Topology 7 (2007), 1579-1598.

[3] D. Ilyutko, Framed 4-Valent Graphs: Euler Tours, Gauss Circuits and Rotating Circuits // arXiv:Math.CO/0911.5504.

[4] D. Ilyutko, V. Manturov, Introduction to graph-link theory // Journal of Knot Theory and Its Ramifications, 18(6) (2009), 791-823.

[5] D. Ilyutko, V. Manturov, Graph links // Dokl. Akad. Nauk 428 (2009), no. 5, 591-594 (Russian).

[6] P. Ozsváth, J. Rasmussen, Z. Szabó, Odd Khovanov homology // www.arxiv.org/math-qa/0710.4300.

[7] L. Traldi, L. Zulli, A bracket polynomial for graphs // arXiv:math.GT/0808.3392.

[8] S.Wehrli, Khovanov homology and Conway mutation // arXiv:math.GT/0301.312. 